\title{
The Role of Innate Immunity in the Pathogenesis of Breast Cancer
}

\author{
Damir Grebića Tamara Gulićb Alma Starčevićc Manuela Alvirovićd \\ Gordana Blagojević Zagorac ${ }^{\mathrm{b}}$ Petra Valković Zujiće \\ Danijela Veljković Vujaklija ${ }^{\mathrm{e}}$ \\ a Department of General and Oncological Surgery, Clinical Hospital Center Rijeka, School of Medicine, University of \\ Rijeka, Rijeka, Croatia; ${ }^{b}$ Department of Physiology and Immunology, School of Medicine, University of Rijeka, \\ Rijeka, Croatia; 'Tissue Typing Laboratory, Clinical Institute for Transfusion Medicine, Clinical Hospital Center Rijeka, \\ Rijeka, Croatia; ${ }^{\mathrm{d} D e p a r t m e n t}$ of Pathology, School of Medicine, University of Rijeka, Rijeka, Croatia; ${ }^{\text {eDepartment of }}$ \\ Radiology, Clinical Hospital Center Rijeka, School of Medicine, University of Rijeka, Rijeka, Croatia
}

\section{Keywords}

Breast cancer · Immunotherapy · Natural killer cells ·

Tumor-associated macrophages · Tumor microenvironment cancer patients. Currently, one of the most used strategies for breast cancer of newly diagnosed patients is neoadjuvant chemotherapy.

\begin{abstract}
Background: Breast carcinoma is the most common malignant disease in the female population and one of the leading causes of death among women worldwide. One crucial hallmark of cancer is chronic inflammation where the immunosuppressive environment is dominant. The immunosuppressive environment is largely achieved by the interaction of tumor cells and infiltrating leukocytes. Summary: Usually, human macrophages and natural killer cells are involved in antitumor immunity. The therapeutic potential of this population against cancers has stimulated their study and led to the discovery of several different tumor-associated macrophages and natural killer cell subsets, each of which is endowed with different immunoregulatory functions. Both heterogeneity and plasticity of the tumor-associated macrophages and natural killer cell compartment, which are both tightly linked to the tumor microenvironment of different breast cancer types. Key Messages: The identification of specific tumor-associated macrophages and natural killer cell subsets endowed with particular functional capabilities might help monitor tumor-mediated responses in breast
\end{abstract}

\section{Introduction}

Breast carcinoma is the most common malignant disease in the female population and one of the leading causes of death among women worldwide. At present, various breast tumor classification systems are utilized; more commonly adopted are the TNM classification and immunohistochemistry classification. Traditional prognostic factors for breast carcinoma include: tumor size, axillary lymph node status, degree of tumor differentiation, and presence of lymph vascular invasion. In addition to the above prognostic factors for breast carcinoma, the treatment method is influenced by the molecular division of breast carcinoma in four subtypes. These include luminal A, luminal B (which can be human epidermal growth factor receptor 2 [HER2]-positive and HER2-negative), HER2-positive, and triple-

Damir Grebić and Tamara Gulić contributed equally to this work. damir.grebic@medri.uniri.hr 
negative cancer. Luminal A has the best prognosis of the listed carcinomas (hormone receptor positive and HER2 negative), while the most malignant type with quick relapse or even visceral disease dissemination is triplenegative cancer (hormone receptor negative and HER2 negative with a high proliferation marker- Ki67) [1]. Besides the prognostic significance of the above division, there is significance for the treatment of this disease. HER2-positive tumors are compulsorily treated with immunotherapy; i.e., using a monoclonal antibody for HER2, but what is new in today's treatment approach is the option for neoadjuvant chemotherapy, which also depends on the given carcinoma subtype. With this in mind, for luminal A subtype, neoadjuvant chemotherapy is not indicated because of its poor effect, while for malignant forms such as HER2-positive and triple-negative tumors, if advanced, neoadjuvant chemotherapy is beneficial and must be included for HER2-positive carcinomas along with immunotherapy (herceptin) $[2,3]$. Determining the benefit of neoadjuvant chemotherapy is problematic for patients with carcinoma subtype luminal B HER2-negative tumors [4, 5]. In these patients, in order to determine the benefit of neoadjuvant chemotherapy, a whole palette of genes must be analyzed using genetic tests. Well-known tests include MammaPrint and OncotypeXD. Today, HER2 positivity is determined not only by immunohistochemistry, but also using molecular biology methods. Cases with weak membrane staining for HER2 protein should be subjected to molecular biology methods to test HER2 gene activity. One of the above-mentioned molecular tests is in situ hybridization. A tumor is considered HER2 positive only if it is concluded that the gene which codes for HER2 protein synthesis is amplified $[4,6]$. The common denominator of the development of cancer and autoimmune/neurodegenerative diseases is chronic inflammation, where the immunosuppressive environment is crucial [7-9]. Growing evidence supports the hypothesis that the local inflammatory processes modulate the microenvironment, which is the key trigger of the molecular etiopatogenetic mechanisms of tumor development [9-11]. Inflammation mediators transform normal cells by activating oncogenes or getting rid of the anti-oncogenic activity, inducing tumor progression of preneoplastic lesions, apoptosis resistance, and differentiation $[9,12]$. The immunosuppressive microenvironment is largely achieved by the action of infiltrating leukocytes, which constitute a large percentage of cellular populations in tumor tissue $[7,12,13]$.

In this review, we focus essentially on tumor-associated macrophages (TAM) and natural killer (NK) cells, providing a summary of the current knowledge of these cells and their possible therapeutic purpose.

\section{The Role of TAM in Breast Cancer}

In the last decade, TAM have been attributed to have a key role in supporting tumor growth and dissemination $[13,14]$. They are heavily involved in cancer-related inflammation $[10,12]$. As a critical component of tumor microenvironment (TME), TAM affect tumor growth, tumor angiogenesis, immune regulation, metastasis, and chemoresistance $[13,14]$. Macrophages commonly represent the highest proportion of myeloid cells in the TME, and their function is mostly associated with pro-tumoral activity [11-3]. High plasticity and variety are the main characteristics of the monocyte lineage [15]. It is clear that macrophages are capable of displaying very different and even opposite phenotypes, depending on the microenvironment they are embedded in $[15,16]$. Activated macrophages are often classified into the M1 (classicalactivated macrophages) and M2 (alternative-activated macrophages) phenotypes [16, 17]. In general, M1 macrophages foster inflammation response against invading pathogens and tumor cells [16], whereas M2 macrophages tend to exert an immune-suppressive phenotype, favoring tissue repair and tumor progression [17, 18]. These two extremes of macrophages are distinct in their different markers, metabolic characteristics, and gene expression profiles. M1 macrophages secrete pro-inflammatory cytokines such as IL-12, tumor necrosis factor- $\alpha$, CXCL-10, and interferon- $\gamma$ and produce high levels of nitric oxide synthase (an enzyme metabolizing arginine to the "killer" molecule nitric oxide) $[15,16]$, while M2 macrophages secrete anti-inflammatory cytokines such as IL-10, IL-13, and IL-4 and express abundant arginase-1, mannose receptor (D206), and scavenger receptors $[17,18]$. Through the production of different cytokines, chemokines, and other factors such as CCL2 and IL-13, tumor cells are able to modulate and polarize macrophage function and characteristics converting them into TAM $[19,20]$. Many studies on genomic and proteomic levels showed that TAM usually display an M2like phenotype [15]. Mantovani et al. [14] determinate that the TAM phenotype (CD $\left.68^{+} / \mathrm{CD} 206^{\text {high }} / \mathrm{CD} 163^{\text {high }}\right)$ contribute to tumor progression at different levels: promoting genetic instability, nourishing tumor stem cells, supporting molecular mechanisms of tumor dissemination, and inhibiting adaptive immunity. In $\mathrm{HER}^{+}$breast cancer, CCL2 expression increases which results in the production of Wnt-1 by intra-epithelial macrophages, inducing the disruption of tumor cell-cell junctions leading to an early dissemination and lung intravasation even before the primary tumor becomes evident $[19,20]$. Moreover, different studies showed that TAM infiltration correlated with poor prognostic breast cancer characteristics such as larger tumor size, higher tumor grade, lymph node metastasis, vascular invasion, hormone receptor 
negativity,HER2 expressions, and basal phenotype [21, 22]. In addition, $\mathrm{ER}^{-}$breast tumors demonstrate a higher infiltration of TAM, which correlates with a lack of pathologically complete response to neoadjuvant chemotherapy and a poorer outcome [22]. Medrek et al. [21] observed that $\mathrm{CD}_{163^{+}}$macrophages in tumor stroma positively correlated with higher grade, larger tumor size, Ki67 positivity, estrogen receptor negativity, progesterone receptor negativity, triple-negative/basal-like breast cancer, and inversely correlated with luminal A breast cancer. Considering these data in general, high infiltration of TAM is associated with an unfavorable outcome and survival in patients with primary invasive breast cancer. Their polarization, localization, and the relative amount related to other types of breast cancer may have important clinical relevance and prognostic impact.

\section{New Insights into Breast Cancer-Targeting Strategies of TAM}

Collectively, many preclinical studies illustrated the pro-tumor function of TAM in breast cancer what makes these cells a good therapeutic target $[23,24]$. Actually, during recent years, a huge effort has been done to develop efficiently drugs able to target TAM, eliminating them from the tumor tissue or even reprogramming their activation state [24]. Several pre-clinical studies indicate that targeting of TAM might be an efficient goal to limit tumor growth and the dissemination process $[23,24]$. One promising agent could be zoledronic acid, which is used to repolarize TAM to the M1 type in breast cancer and other solid tumors [25]. In addition, one strategy could be blocking of the CCL2-CCR2 [26] or CXCL12/ CXCR4 [27] signalling axis to re-educate macrophage polarization toward the pro-tumor phenotype and to block recruitment of TAM by genetic ablation or antibodies.

Many questions still remain open, and we need to extensively study the molecular mechanisms regulating the link between TAM and cancer to identify new prognostic targeting strategies and predictive biomarkers.

\section{The role of NK Cells in Breast Cancer}

NK cells are innate immune cells and play a role in the early immune response in tumors [28, 29]. NK cells respond to inflammatory stimuli and are best known for their role in tumor immunosuppression. Phenotypically, $\mathrm{NK}$ cells are defined as $\mathrm{CD} 56^{+} \mathrm{CD} 16^{+} \mathrm{CD} 3^{-}$in humans $[28,30]$. NK cell activity depends on the interplay of their activating and inhibiting receptor repertoire $[28,30]$. After activation, NK cells secrete proinflammatory cytokines such as interferon- $\gamma$, tumor necrosis factor- $\alpha$, gran- ulocyte macrophage colony-stimulating factor, and chemokines (CCL1, CCL2, CCL3, CCL4, CCL5 and CXCL8) that can modulate the function of other innate and adaptive immune cells $[29,31,32]$. CD94/NK group 2 member A (NKG2A) heterodimer receptor is one of the NK inhibitory receptors that binds to nonclassical minimally polymorphic HLA class I molecules such as HLA-E that is overexpressed in many solid tumors $[29,30]$. In addition, HLA-E expression may represent an important regulatory mechanism of tumors to evade immune surveillance [30]. Mamessier et al. [32] determinate the importance of tolerogenic NK cells in the control of invasive breast tumors resulting in impaired malignant cellular immunogenicity. Crucial receptors for tolerogenic properties of NK cells are the cell Ig-like receptors and/or NKG2A as important factors for the recognition of breast tumor cells [32, 33]. Muraro et al. [34] showed an increased percentage of NK cells in women with HER2 ${ }^{+}$tumors and observed that reduced NK cell infiltration into tumor tissue may be a predictive indicator of failure of chemotherapy treatment in breast cancer. Many questions on the interaction of tumor cells and NK cells are still open. Every day, scientists gain new insights into the function of NK cells in breast cancer. Their functional changes and infiltration localization related to different immunophenotypes of breast cancer may be important clinical prognostic factors.

\section{New Insights into Breast Cancer-Targeting Strategies of NK Cells}

In the last decade, increasing evidence from clinical studies supports the potential efficacy of NK cell therapies in multiple cancers $[35,36]$. NK cells represent an attractive target for cancer immunotherapy owing to their innate capacity to eliminate malignant tumors in a non-major histocompatibility complex (MHC) and non-tumor antigen-restricted manner. NK cell-based immunotherapy has been associated with the treatment of hematologic cancers as well as in patients with breast cancer [37]. The most widely used strategy in cancer immunotherapy is to employ tumor-specific antibodies that promote ADCC through the ligation of CD16 receptors on NK cells. Rituximab (mAb to the B-cell marker CD20), trastuzumab ( $\mathrm{mAb}$ to ErbB2/HER2) and cetuximab ( $\mathrm{mAb}$ to EGFR) have demonstrated marked efficacy in the treatment of various solid and hematological tumors [34]. One of the studies showed that downregulation of NKG2A significantly enhances the anti-tumor capacity of NK cells, so it could be one of the promising therapeutic targets [29]. Another, PD-L1 blockade is known to enhance anti-tumor efficacy of NK cells and may open new lines in immunotherapy with the goal of overcoming im- 
mune suppression in cancer, thereby improving patient outcomes [36]. NK cells have become an important factor in the treatment of cancer. Adaptive NK cell therapy offers great opportunities in the treatment of cancer. Furthermore, NK cells are effective in preventing disease relapse [37]. The decrease in the percentage of NK cells in immune infiltration in tumor tissue may be a predictive indicator of chemotherapy treatment outcome for breast cancer [38].

\section{Conclusion}

The innate immune cell infiltration, in particular TAM and NK cells, exerts essential roles in the development and regulation of tumor growth [39] The expected role of innate immune cells is anti-tumor response against tumor growth; however, data collected over the years demonstrate that cancer cells can modulate the anti-tumoral properties of innate immune cells. TAM acquire an M2like phenotype in the tumor bed and become the major orchestrator for creating an appropriate TME, promote survival of cancer cells, angiogenesis, and cancer cell dissemination. NK cells are less cytotoxic, possibly with immunosuppressive properties facilitating cancer growth.
Finally, accumulating evidence demonstrated that innate immune cells play a pivotal role in the immunosuppressive TME and correlate with tumor progression. All together, these observations strongly suggest that targeting tumor-infiltrating TAM and NK cells represents a promising therapeutic tool against cancer.

\section{Disclosure Statement}

The authors have no conflicts of interest to declare.

\section{Funding Sources}

The investigation was supported by grants from the University of Rijeka (Science Support No. uniri-18.07.2.1.02 to Assist. Prof. D. Grebić, Science Support No. uniri-pr-biomed-19-171498 to Assist. Prof. T. Gulić).

\section{Author Contributions}

Conceptualization, D.G., T.G.; investigation, D.G., T.G., A.S., M.A.; data curation, P.V.Z., D.V.V; writing - original draft preparation, D.G., T.G., A.S; writing - review and editing, G.B.Z., P.V.Z., D.V.V; supervision, D.G.

\section{References}

1 Strehl JD, Wachter DL, Fasching PA, Beckmann MW, Hartmann A. Invasive breast cancer: Recognition of molecular subtypes. Breast Care (Basel). 2011;6(4):258-64.

2 Ellis MJ. Mutational analysis of breast cancer: guiding personalized treatments. Breast. 2013 Aug;22 Suppl 2:S19-21.

3 Mohamed A, Krajewski K, Cakar B, Ma CX. Targeted therapy for breast cancer. Am J Pathol. 2013 Oct;183(4):1096-112.

4 Garraway LA, Verweij J, Ballman KV. Precision oncology: an overview. J Clin Oncol. 2013 May;31(15):1803-5.

5 Ignatiadis M, Sotiriou C. Luminal breast cancer: from biology to treatment. Nat Rev Clin Oncol. 2013 Sep;10(9):494-506.

6 Dedic Plavetić N, Kulic A Vrbanec D. Role of HER-2 signalling pathway in breast cancer: biology, detection and therapeutical implications. Period Biol. 2012;114:505-10.

7 Radolovic P, Grebic D, Mustac E, Sebaher I, Mamic J, Miletić WM. Heat shock protein gp96 and CD4+ and CD8+ T lymphocytes expression as prognostic factors in various molecular types of invasive breast carcinoma. Neoplasma. 2020 Jan:190601N478.

8 Momenimovahed Z, Salehiniya H. Epidemiological characteristics of and risk factors for breast cancer in the world. Breast Cancer (Dove Med Press). 2019 Apr;11:151-64.

9 Hanahan D, Weinberg RA. Hallmarks of cancer: the next generation. Cell. 2011 Mar; 144(5):646-74.
10 Hanahan D, Coussens LM. Accessories to the crime: functions of cells recruited to the tumor microenvironment. Cancer Cell. 2012 Mar;21(3):309-22.

11 Linde N, Casanova-Acebes M, Sosa MS, Mortha A, Rahman A, Farias E, et al. Macrophages orchestrate breast cancer early dissemination and metastasis. Nat Commun. 2018 Jan;9(1):21.

12 Bense RD, Sotiriou C, Piccart-Gebhart MJ, Haanen JB, van Vugt MA, de Vries EG, et al. Relevance of tumor-infiltrating immune cell composition and functionality for disease outcome in breast cancer. J Natl Cancer Inst. 2016 Oct;109(1):1-9.

13 Franklin RA, Liao W, Sarkar A, Kim MV, Bivona MR, Liu K, et al. The cellular and molecular origin of tumor-associated macrophages. Science. 2014 May;344(6186):921-5.

14 Mantovani A, Marchesi F, Malesci A, Laghi L, Allavena P. Tumour-associated macrophages as treatment targets in oncology. Nat Rev Clin Oncol. 2017 Jul;14(7):399-416.

15 Martinez FO, Gordon S, Locati M, Mantovani A. Transcriptional profiling of the human monocyte-to-macrophage differentiation and polarization: new molecules and patterns of gene expression. J Immunol. 2006 Nov; 177(10):7303-11.

16 Mosser DM, Edwards JP. Exploring the full spectrum of macrophage activation. Nat Rev Immunol. 2008 Dec;8(12):958-69.
17 Mantovani A, Sozzani S, Locati M, Allavena P, Sica A. Macrophage polarization: tumor-associated macrophages as a paradigm for polarized M2 mononuclear phagocytes. Trends Immunol. 2002 Nov;23(11):54955

18 Biswas SK, Mantovani A. Macrophage plasticity and interaction with lymphocyte subsets: cancer as a paradigm. Nat Immunol. 2010 Oct;11(10):889-96.

19 Kitamura T, Qian BZ, Soong D, Cassetta L, Noy R, Sugano G, et al. CCL2-induced chemokine cascade promotes breast cancer metastasis by enhancing retention of metastasisassociated macrophages. J Exp Med. 2015 Jun; 212(7):1043-59.

20 Bonapace L, Coissieux MM, Wyckoff J, Mertz KD, Varga Z, Junt T, et al. Cessation of CCL2 inhibition accelerates breast cancer metastasis by promoting angiogenesis. Nature. 2014 Nov;515(7525):130-3.

21 Medrek C, Pontén F, Jirström K, Leandersson $\mathrm{K}$. The presence of tumor associated macrophages in tumor stroma as a prognostic marker for breast cancer patients. BMC Cancer. 2012 Jul;12(1):306.

22 Tymoszuk P, Evens H, Marzola V, Wachowicz $\mathrm{K}$, Wasmer $\mathrm{MH}$, Datta $\mathrm{S}$, et al. In situ proliferation contributes to accumulation of tumor-associated macrophages in spontaneous mammary tumors. Eur J Immunol. 2014 Aug;44(8):2247-62. 
23 DeNardo DG, Brennan DJ, Rexhepaj E, Ruffell B, Shiao SL, Madden SF, et al. Leukocyte complexity predicts breast cancer survival and functionally regulates response to chemotherapy. Cancer Discov. 2011 Jun;1(1): 54-67.

24 Georgoudaki AM, Prokopec KE, Boura VF, Hellqvist E, Sohn S, Östling J, et al. Reprogramming tumor-associated macrophages by antibody targeting inhibits cancer progression and metastasis. Cell Rep. 2016 May; 15(9):2000-11.

25 Gnant M, Mlineritsch B, Stoeger H, LuschinEbengreuth G, Heck D, Menzel C, et al.; Austrian Breast and Colorectal Cancer Study Group, Vienna, Austria. Adjuvant endocrine therapy plus zoledronic acid in premenopausal women with early-stage breast cancer: 62-month follow-up from the ABCSG-12 randomised trial. Lancet Oncol. 2011 Jul; 12(7):631-41

26 Lim SY, Yuzhalin AE, Gordon-Weeks AN, Muschel RJ. Targeting the CCL2-CCR2 signaling axis in cancer metastasis. Oncotarget. 2016 May;7(19):28697-710.

27 Liang Z, Zhan W, Zhu A, Yoon Y, Lin S, Sasaki $\mathrm{M}$, et al. Development of a unique small molecule modulator of CXCR4. PLoS One. 2012;7(4):e34038.
28 Laskarin G, Redzovic A, Vukelic P, Veljkovic D, Gulic T, Haller H, et al. Phenotype of NK cells and cytotoxic/apoptotic mediators expression in ectopic pregnancy. Am J Reprod Immunol. 2010 Nov;64(5):347-58.

29 Kamiya T, Seow SV, Wong D, Robinson M, Campana D. Blocking expression of inhibitory receptor NKG2A overcomes tumor resistance to NK cells. J Clin Invest. 2019 Mar; 129(5):2094-106.

30 Gulic T, Laskarin G, Dominovic M, Glavan Gacanin L, Babarović E, Rubesa Z, et al. Granulysin-mediated apoptosis of trophoblasts in blighted ovum and missed abortion. Am J Reprod Immunol. 2018 Sep;80(3):e12978.

31 Silva GB, Silva TG, Duarte RA, Neto NL, Carrara $\mathrm{HH}$, Donadi EA, et al. Expression of the Classical and Nonclassical HLA Molecules in Breast Cancer. Int J Breast Cancer. 2013;2013: 250435.

32 Mamessier E, Sylvain A, Thibult ML, Houvenaeghel G, Jacquemier J, Castellano R, et al. Human breast cancer cells enhance self tolerance by promoting evasion from NK cell antitumor immunity. J Clin Invest. 2011 Sep; 121(9):3609-22

33 Tu MM, Rahim MM, Sayed C, Mahmoud AB, Makrigiannis AP. Immunosurveillance and Immunoediting of Breast Cancer via Class I MHC Receptors. Cancer Immunol Res. 2017 Nov;5(11):1016-28.
34 Muraro E, Martorelli D, Turchet E, Miolo G, Scalone S, Comaro E, et al. A different immunologic profile characterizes patients with HER-2-overexpressing and HER-2-negative locally advanced breast cancer: implications for immune-based therapies. Breast Cancer Res. 2011;13(6):R117.

35 Tallerico R, Conti L, Lanzardo S, Sottile R, Garofalo C, Wagner AK, et al. NK cells control breast cancer and related cancer stem cell hematological spread. Oncoimmunology. 2017 Feb;6(3):e1284718.

36 Oyer JL, Gitto SB, Altomare DA, Copik AJ. PD-L1 blockade enhances anti-tumor efficacy of NK cells. Oncoimmunology. 2018 Aug; 7(11):e1509819.

37 Garner WL, Minton JP, James AG, Hoffmann CC. Human breast cancer and impaired NK cell function. J Surg Oncol. 1983 Sep;24(1): 64-6.

38 Campbell MJ, Baehner F, O’Meara T, Ojukwu E, Han B, Mukhtar R, et al. Characterizing the immune microenvironment in high-risk ductal carcinoma in situ of the breast. Breast Cancer Res Treat. 2017 Jan;161(1):17-28.

39 Mattiola I, Tomay F, De Pizzol M, SilvaGomes R, Savino B, Gulic T, et al. The macrophage tetraspan MS4A4A enhances dectin1-dependent NK cell-mediated resistance to metastasis. Nat Immunol. 2019 Aug;20(8): 1012-22. 